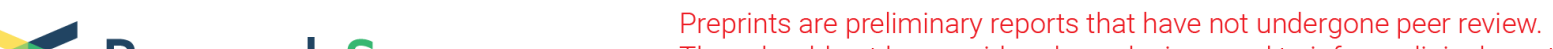 Research Square They should not be considered conclusive, used to inform clinical practice, or referenced by the media as validated information.
}

\section{Dual blockade of IL- 6 and CTLA-4 regresses pancreatic tumors in a CD4+ T-cell-dependent manner.}

Michael Ware

Emory University

Christopher McQuinn

The Ohio State University

Mohommad Zaidi

Emory University Winship Cancer Insittute

Hannah Knochelmann

Emory University Winship Cancer Insittute

\section{Brian Robinson}

The Winship Cancer Institute of Emory University

\section{Cameron Herting}

Emory University

\section{Thomas Mace}

Ohio State University https://orcid.org/0000-0003-1070-6819

\section{Zhengjia Chen}

Emory University

\section{Chao Zhang}

Department of Biostatistics and Bioinformatics Shared Resource, Winship Cancer Institute, Emory University Rollins School of Public Health, Atlanta, GA 30322, USA

\section{Matthew Farren}

Emory University Winship Cancer Insittute

\section{Amanda Ruggieri}

Department of Hematology and Medical Oncology, Winship Cancer Institute of Emory University

\section{Jacob Bowers}

The Ohio State University

\section{Reena Shakya}

Ohio State University

\section{Alton Farris III}

Emory University https://orcid.org/0000-0001-5534-7763

\section{Greg Young}

The Ohio State University 


\section{William Carson III}

The Ohio State University

\section{Bassel El-Rayes}

Emory University

\section{Chrystal Paulos}

Emory University Winship Cancer Insittute

Gregory B. Lesinski ( $\square$ gregory.b.lesinski@emory.edu )

Emory University https://orcid.org/0000-0002-8787-7678

\section{Article}

Keywords: pancreatic tumors, anti-tumor immune responses, pancreatic cancer

Posted Date: June 21st, 2021

DOI: https://doi.org/10.21203/rs.3.rs-599225/v1

License: (c) (1) This work is licensed under a Creative Commons Attribution 4.0 International License.

Read Full License 


\section{Abstract}

This study aimed to enhance anti-tumor immune responses to pancreatic cancer via antibody-based blockade of interleukin-6 (IL-6) and cytotoxic T-lymphocyte-associated protein 4 (CTLA-4). Mice bearing subcutaneous MT5 or orthotopic KPC-luc pancreatic tumors were treated with antibodies to IL-6, CTLA-4, or the combination. This combination significantly inhibited tumor growth, accompanied by overwhelming T-cell infiltration. Tcell depletion studies unveiled a unique dependence on $\mathrm{CD} 4^{+} \mathrm{T}$-cells for anti-tumor activity of this combination therapy. In vivo blockade of CXCR3 prevented orthotopic tumor regression in the presence of the combination treatment, demonstrating an integral role for the CXCR3 axis in mediating efficacy. Increased expression of CXCR3 on tumor infiltrating cells was observed by IHC and by PCR. These data represent the first report of IL- 6 and CTLA- 4 blockade as a means to regress pancreatic tumors with defined operative mechanisms of efficacy. Given these results, this therapeutic combination has potential for immediate clinical translation.

\section{Introduction}

Antibodies targeting immune checkpoint receptors and their ligands have gained regulatory approval and demonstrated efficacy in patients. The most notable examples include blockade of cytotoxic Tlymphocyte protein-4 (CTLA-4) and programmed cell death protein 1 (PD-1). Despite encouraging data in patients with various malignancies, there remain a number of key challenges with this approach ${ }^{1}$. Many patients still do not benefit from immune checkpoint inhibition (ICI), while resistance is common in those who do initially respond to therapy ${ }^{1}$. Most pancreatic tumors are also inherently resistant to $\mathrm{ICl}^{2}$. Overcoming these limitations is a priority and could advance outcomes across multiple tumor types.

The desmoplastic stroma unique to PDAC is a dynamic, immune suppressive component contributing to the limited impact of immune therapy in this malignancy. Our laboratory and others have demonstrated that PDAC stroma and stromal-derived cytokines and chemokines restrain host immunity ${ }^{3-7}$. Although dysregulated cytokines represent rational targets, there are limited data to prioritize them in patients. In a cohort of seventy-two treatment naïve patients with metastatic PDAC, interleukin-6 (IL-6) correlated with reduced overall survival ${ }^{8}$. These data were intriguing, as IL-6 can regulates phenotypic and functional properties of various lymphocyte and myeloid cell populations ${ }^{9}{ }^{10}$. Detailed immune phenotyping of peripheral blood cells from the same patient cohort revealed a similar relationship between reduced overall survival and elevated circulating T-cells expressing CTLA-4 ${ }^{8}$.

These data encourage strategic combination therapies incorporating CTLA-4-targeted antibodies in PDAC. Based on these data, we hypothesized that IL- 6 blockade would enhance efficacy of anti-CTLA-4 therapy. Herein, we report that combined blockade of IL- 6 and CTLA-4 inhibits PDAC growth by potentiating infiltration of T-cells into tumors. Notably, this therapy relies on CD $4^{+}$T-cells for its efficacy, while in vitro it promotes IFN-y production by activated antigen-specific $\mathrm{CD} 4^{+} \mathrm{T}$-cells. Aligned with these observations, we demonstrate PDAC cells produce lymphocyte-attracting CXCR3-associated chemokines 
in response to IFN-y stimulation, including high levels of the CXCR3-associated chemokine CXCL10. In vivo studies further indicate systemic and intratumoral increases in CXCR3-expressing CD ${ }^{+} \mathrm{T}$ cells after dual IL-6/CTLA-4 blockade and antitumor efficacy that is dependent upon CXCR3. Finally, this treatment induced systemic increases in $\mathrm{CD} 4^{+}$T-helper subsets expressing TBET and GATA3. Together our findings suggest combined blockade of IL- 6 and CTLA- 4 can regress pancreatic tumors via a unique mechanism by imparting $\mathrm{CD} 4^{+} \mathrm{T}$-cell-mediated anti-tumor immunity.

\section{Results}

Combined blockade of IL-6 and CTLA-4 augments antitumor efficacy in murine PDAC models.

We first determined efficacy of dual IL- 6 and CTLA-4 blockade in mice bearing subcutaneous MT5 tumors. The MT5 cell line originated from a KPC tumor, harboring G12D mutated Kras and R172H mutated Trp53 ${ }^{11}$. Tumor growth was significantly reduced in mice treated with combined IL-6 and CTLA4 blocking antibodies compared to mice treated with isotype control antibodies $(p=0.0001)$, single agent CTLA-4 blockade $(p=.0207)$, or IL-6 blockade $(p=.0002)$ (Fig. 1A). Although anti-CTLA-4 alone inhibited tumor growth to a greater extent than mice treated with an isotype control $(p=0.0004)$, blockade of IL-6 alone did not delay tumor growth. This impact of dual IL- 6 and CTLA-4 blockade on tumor growth was encouraging, however the mechanism required further investigation.

Combined IL-6 and CTLA-4 blockade increases T-cells in pancreatic tumors.

In light of previous research investigating the influence of IL-6 $6^{12}$ and CTLA-4 ${ }^{12-14}$ on T-cells, we hypothesized combined blockade would increase T-cell infiltration into pancreatic tumors. Immunohistochemical staining of tumors from mice treated with this therapy indicated increased T-cells within the tumor compared to mice receiving isotype control or single agent therapies (Fig. 1B-C). Image quantification revealed both single agent blockade of IL-6 $(p=0.0038)$ or CTLA-4 $(p=0.0035)$ increased $\mathrm{CD}^{+}{ }^{+}$T-cell infiltration versus isotype control-treated mice (Fig. 1B). Mice given combined therapy had more T-cells infiltrating tumors compared to mice treated with either single agent blockade of IL-6 $(p=$ $0.035)$, CTLA-4 ( $p=0.038)$ or isotype controls $(p=0.0001)$ indicating a superior effect of IL-6 and CTLA-4 blockade on T-cell infiltration in this tumor model (Fig. 1B).

Systemic changes in Th1 immunity occur following IL-6 and CTLA-4 blockade.

The role of IL- 6 in regulating differentiation of T-cell subsets has been documented ${ }^{15-17}$, while evidence suggests CTLA-4 blockade promotes differentiation of Th2 and T follicular (Tfh) subsets ${ }^{12} 1819$. Therefore, we evaluated the impact of this combination treatment on splenic T-cells as a surrogate of systemic changes. Anti-CTLA-4 alone or in combination with IL- 6 blockade increased circulating cells with a Th1 phenotype $\left(\mathrm{CD} 4^{+} \mathrm{CCR} 6^{-} \mathrm{CXCR} 3^{+} \mathrm{CCR} 4^{-}\right)$as compared to mice treated with isotype control $(\mathrm{p}<$ 0.0001 and $p=0.0004$, respectively) or anti-IL-6 alone ( $p=0.0026$ and $p=0.0268$, respectively) (Fig. 1DE). No change in cells with Th2 $\left(\mathrm{CD} 4^{+} \mathrm{CCR} 6^{-} \mathrm{CXCR}^{-} \mathrm{CCR} 4^{+}\right)$or Th17 $\left(\mathrm{CD} 4^{+} \mathrm{RORYt}^{+}\right)$phenotypes were evident 
(Fig. 1D-E and Supplementary Fig. 1A-B, respectively). Unexpectedly, the proportion of splenocytes expressing T-regulatory cell (Treg) markers $\left(\mathrm{CD} 4^{+} \mathrm{CD} 25^{+} \mathrm{FoxP} 3^{+}\right)$was higher in the combination group versus both isotype control $(p=0.0027)$ and anti-IL-6 $(p=0.0007)$ (Supplemental Fig. 1C-D). Because IL6 also regulates expansion of myeloid-derived suppressor cells in PDAC ${ }^{20}$, phenotypic properties of these cells were assessed. No significant change in frequency of either monocytic (CD11b+Ly6G ${ }^{-}$Ly $\left.6 C^{+}\right)$or granulocytic (CD11 b ${ }^{+}$Ly6 $\mathrm{G}^{+} \mathrm{Ly} 6 \mathrm{C}^{\text {low }}$ ) populations were observed in splenocytes across groups (Supplemental Fig. 1E-F). These data indicate a dual role for combined IL6 and CTLA-4 blockade in driving tumoral T-cell infiltration and systemically shifting T-helper phenotypes.

Combined IL-6 and CTLA-4 blockade is dependent upon CD4 ${ }^{+} T$ cells.

Given the increased infiltration of $\mathrm{CD}^{+}{ }^{+}$-cells into subcutaneous tumors and systemic alteration of $\mathrm{CD} 4^{+}$ T-cells, we questioned whether efficacy might be dependent upon $\mathrm{CD} 4^{+}$or $\mathrm{CD} 8^{+} \mathrm{T}$-cells. For these studies, we used a more physiologically-relevant model in which luciferase-expressing KPC-luc cancer cells were orthotopically implanted into the pancreas of immune-competent mice. These cells express enhanced firefly luciferase that allows longitudinal bioluminescent imaging (BLI) of tumors. $\mathrm{CD} 4^{+}$or $\mathrm{CD} 8^{+} \mathrm{Tcells}^{\mathrm{s}}$ were depleted in mice bearing orthotopic KPC-luc tumors prior to treatment with isotype control antibodies, or combined IL-6 and CTLA-4 blockade (Fig. 2A). We confirmed CD4 or CD8 depletion by flow cytometric analysis of cells from the spleens at endpoint (Fig. 2B). Longitudinal BLI data indicated CD $4^{+}$ T-cell-dependent efficacy of combined IL- 6 and CTLA-4 blockade, as CD $4^{+} T$ cell depleted mice receiving therapeutic antibodies had accelerated progression compared to mice receiving only combination therapy (Fig. 2C-D). Tumor progression in some animals was striking, and faster than in mice receiving isotype control antibodies. $\mathrm{CD}^{+}{ }^{+} \mathrm{T}$-cell depletion also impacted tumor growth, albeit not to the magnitude of $\mathrm{CD} 4^{+}$ T-cell depletion, nor to a significant degree compared to combination treated mice (Fig. 2C-D). To complement trends from BLI data, total pancreas and tumor weight was measured post-mortem (Fig. 2E). All mice had primary tumors localized to the pancreas tail, while mice with advanced disease had tumor extensions into the peritoneum. One mouse receiving dual IL-6/CTLA-4 had no evidence of tumor, but pancreas weight was recorded. These data confirmed efficacy in multiple murine PDAC models and highlighted a unique requirement for $\mathrm{CD} 4^{+} \mathrm{T}$-cells.

Combined IL-6 and CTLA-4 blockade supports Th1 cytokines that cross-talk to facilitate chemokine production from tumor cells.

Our observations of greater systemic Th1 cells following combined blockade of IL- 6 and CTLA-4 (Fig. 1E) prompted us to consider direct effects of therapy on cytokine profiles in T cells. To address this, we used an antigen-specific system with transgenic TRP- 1 CD $4^{+}$T-cells. These cells bear TCR recognizing tyrosinase-related protein (TRP-1) to model recognition of endogenous tumor antigen. Consistent with findings in the MT5 model, expanding TRP- 1 CD $4^{+}$T-cells with IL- 6 and CTLA-4 blocking antibodies fostered their capacity to secrete IFNY when re-stimulated with cognate antigen (Fig. 3A-B). This work suggests dual blockade imparts direct effects on $\mathrm{CD} 4^{+} \mathrm{T}$-cells, resulting in Th1-associated cytokines. 
Previous research also suggests IL-6 alters classical IFNy response genes, including chemokines for T cell trafficking ${ }^{21}$. To define how IL- 6 and IFNy-induce chemokines by PDAC cells, we surveyed chemokine production from murine MT5 or KPC-Luc tumor cells following in vitro stimulation with IFNY, IL-6 or both. Supernatants were analyzed by chemokine array and results confirmed by ELISA (Fig. 3C-G and Supplemental Fig. 2A-E). IFNY induced upregulation of canonical chemokines including CXCL10 and CXCL9, which ligate CXCR3 receptor ${ }^{22-25}$. CCL5 was also upregulated by IFN-Y (Fig. 3C-G). ELISA data, revealed no change in chemokines after treatment of MT5 or KPC-luc cells with IL- 6 alone, nor did combined stimulation with IL-6 and IFN- $\gamma$ differ significantly from IFN- $\gamma$ alone as quantified by ELISA (Fig. $3 \mathrm{E}-\mathrm{G})$.

We hypothesized Th1 cell trafficking into tumors via CXCR3 is a key mechanism contributing to efficacy of this therapy. To examine this, we stained tumors for DAPI and CXCR3 and analyzed them by IHC (Fig. 4A). Tumors from mice receiving combined IL- 6 and CTLA- 4 blockade, had increased CXCR3 ${ }^{+} T$ cells as a percentage of total cells, compared to mice receiving isotype control antibodies (Fig. 4B). A subsequent study was conducted to validate whether tumor infiltrating lymphocytes (TIL) from mice expressed elevated CXCR3 in response to dual IL-6/CTLA-4 blockade. Here, CD3 ${ }^{+}$TIL were enriched by bead-based negative selection for $\mathrm{CD} 3^{+}$cells, followed by $\mathrm{CD} 45^{+}$selection to enrich $\mathrm{CD} 3^{+} \mathrm{CD} 45^{+}$cells from established orthotopic tumors after 14 days of treatment with the combination or isotype control antibodies. Endpoint tumor weight and changes in BLI are in Supplemental Fig. 3A-B. Since enzymatic digestion cleaves surface CXCR3, we evaluated RNA transcripts from TIL by PCR. These data revealed significantly greater $\mathrm{CXCR} 3$ in $\mathrm{CD}^{+}$TIL from mice treated with the combination (Fig. 4C). No significant difference in IFN- $y$ transcripts were observed in these TIL (Fig. 4D). Analysis of splenocytes from these same animals showed a greater percentage of circulating $\mathrm{CD} 3^{+} \mathrm{CD} 4^{+} \mathrm{CXCR} 3^{+}$cells, and a greater MFI for CXCR3 on $\mathrm{CD} 45^{+} \mathrm{CD}^{+} \mathrm{CD} 4^{+}$cells (Fig. 4F-G). In contrast, no significant difference in CXCR3 was observed on $\mathrm{CD}^{+}$splenocytes from each treatment group (Fig. $4 \mathrm{H}-\mathrm{J}$ ).

CXCR3 dependent efficacy of combined IL-6 and CTLA-4 blockade. To determine if CXCR3 receptor interactions were essential for the T-cell response, we employed CXCR3 blocking antibodies in mice receiving combination therapy. All mice receiving combined IL- 6 and CTLA-4 blockade experienced tumor regression by BLI (Fig. 4K and Supplemental Fig. 3C). Mice receiving combination therapy demonstrated significant decreases in $\mathrm{BLI}$ signal compared to mice receiving isotype control antibodies, or blockade of IL6 or CTLA-4 alone. Concurrent CXCR3 blockade significantly inhibited efficacy of combination therapy, leading to results similar to treatment with isotype controls (Fig. 4K-L). No significant change in tumor growth by BLI was evident between mice treated with isotype controls or single agent therapy. Postmortem pancreas/tumor weight at study endpoint confirmed growth inhibitory effects of the combination were significant, as compared to treatment with anti-CTLA-4 or isotype controls $(p=0.0778$ and $p=$ 0.0005 , respectively; Fig. $4 \mathrm{~L}$ ). Similar to prior studies (Fig. 2C-E), primary tumor localized to the pancreas tail was confirmed post-mortem, and mice with advanced disease had small peritoneal extensions. In this study, 4 mice in the combination therapy group had no tumors, but pancreatic weight was recorded. 
Notably, this CXCR3-dependent mechanism of concurrent IL-6 and CTLA-4 blockade was confirmed in mice bearing subcutaneous MT-5 tumors (Supplemental Fig. 3D).

Dual blockade therapy promotes CD4 ${ }^{+}{ }^{T-c e l l}$ infiltration of tumors in an orthotopic PDAC model.

Changes in the tumor microenvironment that may explain efficacy of this combination were surveyed by IHC. Although prior observations show IL-6 is derived from fibroblasts in PDAC ${ }^{20} 26$, alpha smooth muscle actin (a-SMA) staining was not significantly different between groups (Supplemental Fig. 4A-B). A trend toward increased $\mathrm{CD} 8^{+} \mathrm{T}$-cells in tumors from mice receiving dual blockade was seen, but not significant (Fig. 5A-B). Although no differences in $\mathrm{CD}^{+}{ }^{+} \mathrm{FoxP}^{+}{ }^{+}$T-cells emerged (Supplemental Fig. 4C), a consistent increase in $\mathrm{CD} 4^{+}$T-cells lacking FOXP3 expression was present in tumors from mice receiving combined IL- 6 and CTLA-4 blockade compared to controls $(p=0.0297)$ or to mice receiving anti-IL-6 alone $(p=$ 0.0439) (Fig. 5C-D).

Dual blockade therapy expands systemic TBET ${ }^{+}$and GATA3 $C D 4^{+}$T-cells in an orthotopic PDAC model.

We next investigated effects of treatment on systemic T-cell phenotypes in the orthotopic model. Flow cytometry (Fig. 6A) recapitulated observations of Th1 immunity, consistent with splenocyte data in the subcutaneous model (Fig. 1D-E). Robust expansion of $\mathrm{CD}^{+}{ }^{+} \mathrm{CD}^{+}{ }^{+} \mathrm{TBET}^{+}$(Th1) T-cells was observed in splenocytes from mice receiving combined IL- 6 and CTLA-4 blockade $(p=0.0015)$ or anti-IL- 6 alone $(p=$ 0.0041 ) versus isotype-treated mice (Fig. 6A). We also observed a significantly higher splenic $\mathrm{CD}^{+}{ }^{+} \mathrm{CD} 4^{+} \mathrm{GATA}^{+}$(Th2) T-cells in mice treated with the combination compared to isotype controls $(p=$ 0.0003; Fig. 6B). Of note, $\mathrm{CD}^{+}{ }^{+} \mathrm{CD} 4^{+} \mathrm{GATA} 3^{+} \mathrm{T}$-cells were more abundant in mice receiving combination blockade, versus those treated with anti-CTLA-4 alone $(p=.0012$; Fig. $6 \mathrm{~B})$. Thus, a unique increase in both $\mathrm{TBET}^{+}$and $\mathrm{GATA}^{+} \mathrm{CD}^{+}{ }^{+}$cells was observed in mice treated with combined IL- 6 and CTLA-4 blockade. We also observed no difference in frequency of T-regulatory cells, defined phenotypically as $\mathrm{CD}^{+} \mathrm{CD} 4^{+} \mathrm{CD} 25^{\text {hi }}{ }^{\mathrm{FOXP}} 3^{+}$, or in $\mathrm{CD} 3^{+} \mathrm{CD} 4^{+} \mathrm{RORYt}^{+}$cells from mice receiving combined IL- 6 and CTLA-4 blockade as compared to isotype control-treated mice (Fig. 6C-D). There were no differences in these systemic biomarkers between mice receiving only the combination or the combination together with CXCR3-targeted antibodies (Fig. 6A-D).

We observed a higher percentage of CD4 ${ }^{+}$T-cells positive for PD- 1 in splenocytes from mice treated with anti-IL-6 alone or combined with CTLA-4 blockade compared to isotype control or single agent anti-CTLA4 (Supplemental Fig. 5A). Contrasting data for $C D 4^{+}$T-cell subsets, few changes were observed in composition of splenic $\mathrm{CD} 8^{+} \mathrm{T}$-cells of mice treated with single agent or combination therapy. However, mice receiving combined IL- 6 and CTLA- 4 blockade demonstrated significantly more CD $8^{+}$PD- $1^{+}$T-cells than mice receiving isotype control or anti-IL-6 antibodies (Supplemental Fig. 5B). Immunologically, this strategy impacted $\mathrm{CD} 4^{+}$T-cells, driving increases in both Th1 and Th2 immunity, with more limited systemic changes in $\mathrm{CD} 8^{+} \mathrm{T}$-cells. 


\section{Discussion}

Antibodies that neutralize IL- 6 have not been effective as single agents in PDAC patients ${ }^{27}$. Moreover, blockade of CTLA-4 has limited efficacy as a single agent drug in this aggressive disease ${ }^{28} 29$. Here, we demonstrate that combined blockade of these targets elicits potent anti-tumor activity in three different pancreatic tumor models: subcutaneous MT5, orthotopic KPC-luc and orthotopic Panc02 pancreatic tumors (Fig. 1A, 2D-E, 4K-L, and Supplemental Fig. 6A). The efficacy of concurrently targeting IL-6 and immune checkpoints was first established by our group ${ }^{26}$. In these studies, blockade of IL-6 and PD-L1 in MT5 and Panc02 murine PDAC models significantly inhibited tumor growth, while promoting effector $\mathrm{CD}^{+} \mathrm{T}$ cell infiltration of tumors ${ }^{26}$. This effect has been reproduced in murine models including glioblastoma, colorectal cancer and melanoma ${ }^{30-32}$. The data from this report are important in lending flexibility to clinical translation, whereby multiple immune checkpoint antibodies may have efficacy via non-overlapping mechanisms. This study highlights novel immunomodulatory mechanisms observed upon neutralizing IL-6 alongside CTLA-4 blockade, that are distinct from PD-1/PD-L1 blockade.

Results from this study indicate a unique mechanism of action when compared to combined IL- 6 and PD1/PD-L1 blockade. Previously, efficacy of dual IL- 6 and PD-1/PD-L1 blockade was dependent on CD $8^{+}$but not $\mathrm{CD} 4^{+} \mathrm{T}$ cells. While $\mathrm{CD} 8^{+} \mathrm{T}$ cell depletion significantly restored tumor growth in mice receiving dual IL6/CTLA-4 blockade, CD4 ${ }^{+}$depletion resulted in more pronounced tumor growth. Furthermore, in vivo CXCR3 blockade revealed anti-IL-6/CTLA-4 combination therapy was reliant on this chemokine-receptor interaction. Additionally, combined IL- 6 and PD-1/PD-L1 blockade reduced aSMA ${ }^{+}$stromal content, while these changes were not observed following CTLA-4 and IL- 6 blockade ${ }^{26}$. This discrepancy may be due to differential effects on IL-6 producing fibroblast subsets from therapy 3334 .

Previous studies investigating T-cell responses to PDAC propose differing results with respect to the effect of CTLA-4 on CD4 ${ }^{+}$or $\mathrm{CD} 8^{+}$T-cells ${ }^{14}{ }^{35}$. Here we observed a dependence on CD $4^{+}$T-cells to mediate the anti-tumor effects of combined IL- 6 and CTLA-4 blockade. CD4 ${ }^{+}$T-helper support of CD8 cells may provide heterogeneous T-cell responses that mediate PDAC regression. Recent evidence describes the need for $\mathrm{CD}^{+}$and $\mathrm{CD} 8^{+}$T-cell activation in anti-tumor responses ${ }^{36}$. This study reinforces the importance of $\mathrm{CD} 4^{+}$T-cells in optimal efficacy of immunotherapy.

We hypothesized efficacy of IL- 6 and CTLA-4 blockade may be mediated in part by tumor-derived chemokines that enhance lymphocyte trafficking into tumors. Studies of anti-CTLA-4 alone in murine PDAC models report presence of tumoral CD $4^{+}$T-cell infiltration from lymph nodes ${ }^{14}$. Our in vitro studies demonstrate combined IL- 6 and CTLA-4 blockade elicits increases in IFNy producing CD $4^{+}$T-cells in the context of antigen specific activation ${ }^{12}{ }^{13}$. While we observed this effect may be driven by blockade of IL6 , previous studies in prostate cancer observed elevated numbers of IFNY producing CD $4^{+} \mathrm{T}$ cells upon administration of CTLA-4 blocking antibodies. IFNY is appreciated as a multifaceted soluble factor capable of directly inhibiting tumor cell growth, driving immune activation, and stimulating production of interferon response genes by tumor cells ${ }^{37-40}$. One group has demonstrated the activation of interferon 
response genes in cancer cells occurs, suggesting this may contribute to efficacy of CTLA-4 blockade, such that CTLA-4 blockade fails in patients with defects in these genes ${ }^{41}$. While MT5 and KPC-luc lines used in this study secrete CXCR3 ligands CXCL10 and MIG, it is uncertain if patients with defects in these pathways would benefit from dual blockade of IL-6 and CTLA-4. Indeed, recent reports support importance of the CXCR3 chemokine axis for mediating responses to $\mathrm{ICl}{ }^{42}$, supporting our observations here. Our in vivo studies depleting CD4 ${ }^{+}$T-cells and employing CXCR3 blocking antibody highlight infiltration of CXCR3 expressing T-cells as a likely mechanism mediating tumor regression in the context of this combination therapy.

While this therapy elicits anti-tumor activity, ICI has potential for toxicity in patients. Attempts to ameliorate the autoimmune toxicities of ICl including anti-CTLA-4 and anti-PD-1/PD-L1-targeted antibodies revealed the IL-6R blocking antibody tocilizumab is effective in patients refractory to steroids 4344 . A recent report demonstrated tocilizumab alongside pembrolizumab in melanoma prevented exacerbation of Crohn's disease, and allowed durable anti-tumor immune responses ${ }^{43}$. Similarly, a case report showed tocilizumab in a patient with pulmonary adenocarcinoma completely resolved immunerelated toxicities to nivolumab including oropharyngeal mucositis and esophagitis ${ }^{45}$. Finally, use of IL6Rblocking antibodies with chimeric antigen receptor T-cells (CART) produced encouraging results while enhancing patient safety ${ }^{44}$. This emerging use of IL-6/IL-6R blockade to limit ICl-associated toxicities has led to clinical trials exploring use of IL- 6 blockade for improved safety of these therapies (NCT03601611).

Despite these encouraging results, efforts to apply IL-6 or IL-6R blockade prospectively with therapeutic intent in clinical trials have lagged behind pre-clinical data, possibly due to resistance to repurpose these drugs from autoimmunity into the oncology setting. To date, only a limited number of clinical trials (NCT04191421, NCT04258150) are combining IL-6 blockade therapy with ICI in PDAC. Continued experience with IL- 6 and ICl combinations across solid tumors will inform the field regarding efficacy and ability to limit autoimmune sequelae.

\section{Materials And Methods}

\section{Cell lines and antibodies}

Murine MT5 (Kras ${ }^{L S L-G 12 D}$, Trp53 ${ }^{L S L-R 270 H}$, Pdx1-cre) pancreatic cells were from David Tuveson (Cold Spring Harbor Laboratory, Cold Spring Harbor, NY) and were cultured in RPMI with 10\% FBS and Antibiotic:Antimycotic Solution (GeminiBio). Murine KPC-luc (Kras ${ }^{\mathrm{LSL}-\mathrm{R} 270 \mathrm{H}}, \mathrm{p} 53^{-/-}, \mathrm{Pdx} 1$-cre) cells were from Dr. Craig Logsdon (MD Anderson). Panc02 was provided by Dr. Shari Pilon-Thomas (H. Lee Moffitt Cancer Center, Tampa, FL). KPC-luc and Panc02 cells were cultured in DMEM with $10 \%$ FBS and Antibiotic:Antimycotic Solution. All cell lines were confirmed to be mycoplasma free by both internal and external mycoplasma testing. Murine antibodies to IL-6 (Clone MP5-20F3), CTLA-4 (Clone 9D9), CXCR3 (Clone CXCR3-173) or isotype controls (Clones LTF-2 for subcutaneous or HRPN for orthotopic studies, 
MCP-11 and polyclonal Armenian hamster lgG, respectively) were from BioXcell (West Lebanon, NH). Anti-mouse CD8a (Clone 2.43) and anti-mouse CD4 (Clone GK1.5) depleting antibodies were from BioXcell.

\section{In vivo efficacy studies}

Animal studies were conducted under institutional animal care and use committee (IACUC) approval at The Ohio State University or Emory University. $1 \times 10^{6}$ MT5 tumor cells were injected subcutaneously in the flank of female C57BL/6 mice. Once tumors reached 50-100 mm³ (typically 7-10 days), antibody treatment was initiated. Subcutaneous studies ended once tumors reached volumes meeting IACUCmandated early removal. For orthotopic studies, 6-8 week old female C57BL/6 mice underwent inhalation anesthesia with isoflourane. The abdomen was prepared, draped and a midline laparotomy was made in the upper abdomen. Fascia was incised and spleen mobilized from the left upper quadrant and elevated to identify the pancreas tail. A 27 1/2 gauge needle was used to inject $2 \times 10^{5} \mathrm{KPC}$-luc cells into the anterior medial body of the pancreas. Hemostasis was ensured, the spleen was returned to normal anatomic location and fascia closed with running absorbable suture. Mice were awakened and monitored for one hour. Tumors grew for 7 days prior to randomization and confirmed by bioluminescent imaging (BLI) using the IVIS Spectrum In Vivo Imaging system and luciferase. Mice were then randomized to treatment groups based on BLI. For all studies, antibodies to IL-6, CTLA-4 or CXCR3 were delivered by intraperitoneal injection thrice weekly every 2-3 days with a maximum number of 6 doses per mouse delivered throughout the course of treatment. For depletion studies, CD4 or CD8 depleting antibodies were delivered at day $-3,-1,+1,+3$ and +7 relative to tumor implantation. Following confirmation of tumor establishment by BLI, depleting antibodies were delivered twice weekly every 3-4 days until study endpoint.

\section{Antibodies for flow cytometry and immunohistochemical staining}

Antibodies, with clone names, used for flow cytometry and immunohistochemistry are in Supplementary Table 1.

\section{Flow cytometry}

At completion of subcutaneous efficacy studies, tissues were harvested for immunophenotypic analyses of splenocytes and single cell suspensions from tumors were assessed by flow cytometry as described ${ }^{26}$. Analysis was performed on a LSRII flow cytometer (BD Biosciences) or a Cytek Aurora (Cytek).

Splenocytes from mice bearing orthotopic tumors were stained with Ghost 780 dye to detect live cells and antibodies for CD4 and CD8 T-cell phenotypes. Intracellular staining was performed using the eBioscience Foxp3/Transcription Factor Staining Buffer Set per manufacturer's protocol.

\section{Immunohistochemical analysis}

Formalin fixed paraffin-embedded (FFPE) tumors from subcutaneous experiments underwent IHC analysis following staining with Ab against CD3 (Catalog A0452; Dako). 40x magnification images of tumors (10 images/tumor) were captured using PerkinElmer's Vectra multispectral slide analysis system. inForm software quantified CD3-positive cells (Fast Red chromogen) within each image. Additional slices 
were stained for CD8, and aSMA and scanned with an Olympus Nanozoomer whole slide scanner and analyzed using Qupath (CD8) or FIJI (NIH) for aSMA. Orthotopic tumors were also FFPE. Dual stains for DAPI (Perkin Elmer) with CD4 and FOXP3 were performed using a Roche autostainer and detected with Opal 520 and Opal 630-conjugated secondary (Perkin Elmer), respectively. Slides were imaged using the Vectra Multispectral Imaging System version 2 (Perkin Elmer). Filter cubes for imaging were DAPI (440$680 \mathrm{~nm})$, FITC (520 nm-680 nm), Cy3 (570-690 nm), Texas Red (580-700 nm) and Cy5 (670-720 nm). Multispectral images were analyzed with Qupath ${ }^{46}$.

\section{TRP-1 transgenic CD4 ${ }^{+}$T-cell activation}

CD4 ${ }^{+}$TRP-1 transgenic T-cells ${ }^{47}$ were activated with TRP-1106-130 peptide (SGHNCGTCRPGWRGAACNQKILTVR) loaded at $1 \mu \mathrm{M}$ concentration onto irradiated B6 splenocytes (10Gy) at a 2:1 TRP-1:feeder cell ratio. TRP-1 cells were cultured with monoclonal antibodies targeting CTLA-4

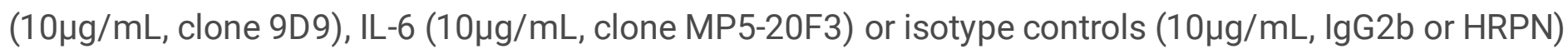
with IL-2 (100IU/mL). Cells were assessed three days after activation for cytokine production post PMA/Ionomycin stimulation. Briefly, cells were activated in PMA (30nM) and lonomycin (20nM) (Sigma) with Monensin $(2 \mu \mathrm{M})$ and Brefeldin $A(5 \mu \mathrm{g} / \mathrm{mL})$ (Biolegend) for 4 hours, followed by fixation and permeabilization for cytokine staining per protocol (BioLegend).

\section{In vitro evaluation of chemokine production}

KPC-luc or MT5 cells were plated at $2 \times 10^{5}$ cells per well in 6 well plates. Media was supplemented with $10 \mathrm{ng} / \mathrm{ml}$ IL-6 (PeproTech), 1 $\mathrm{gg} / \mathrm{ml}$ IFNy (PeproTech), both cytokines combined, or vehicle for 24 hours. Supernatant was collected and spun at $1000 \mathrm{xg}$ then transferred to new tubes to limit cellular contamination. Supernatants were analyzed using Proteome Profiler Mouse Chemokine Array Kits (ARY020, R\&D Systems). Results were confirmed using DuoSet ELISA kits (R\&D Systems) for CXCL10, CXCL9, and CCL5.

\section{Statistical Analysis}

Data from subcutaneous studies obtained by flow cytometry, IHC and tumor volumes were logtransformed prior to analysis to meet model assumptions of normality and homoscedasticity. Tumor volume was modeled over time using mixed-effects regression with fixed effects for group, time and interaction of the two. Random intercepts and slopes by mouse were included with an unstructured covariance matrix for random effects. Other outcomes were compared using ANOVA. P-values $<0.05$ were significant.

For numeric covariates, mean and standard deviation were calculated and presented. One-way ANOVA was performed for $\mathrm{IHC}$ and splenocyte data with univariate analysis. Least significant difference method (LSD) was used for pairwise multiple comparisons. Natural log transformation of bioluminescent imaging data was performed to achieve approximately normal distribution of data. For log transformed data, linear mixed models tested for significant change over time of each outcome and to detect significant difference of each outcome among treatments. Significance was set at 0.05 . For in vitro data, natural log transformation was performed to normally distribute data. We then performed one-way 
ANOVA and LSD to detect whether means significantly differed among treatment groups. All analyses were conducted in SAS v9.4 (SAS Institute, Cary, NC). For experiments where statistical significance was detected, two-tailed t-tests were then utilized to determine pairwise significance

\section{Declarations}

Funding: Supported by NIH grants R01CA208253, R01CA228406 and P30CA138292. The content is solely the responsibility of the authors and does not necessarily represent the official views of the National Institutes of Health.

Contributor Statement: MBW, CM, TAM, CMP and GBL conceptualized and designed experiments. MBW, $\mathrm{CM}, \mathrm{MYZ}$ carried out the animal studies. MBW performed the majority of the in vitro and post in vivo analysis (flow cytometry, IHC). HK, BR, CJH, MRF, ANR and JB contributed to in vivo and in vitro experiments throughout the course of the study. $A B F, R S, W C I I I$, and $B E-R$ provided guidance and consultation on study design and manuscript formation. ZC and CZ performed statistical analysis and provided guidance on powering and designing experiments. MBW, CM, MYZ and GBL drafted the manuscript. All authors contributed to writing and drafting of the final manuscript.

\section{Competing interests:}

Dr. Lesinski has consulted for ProDa Biotech, LLC and received compensation. Dr. Lesinski has received research funding through a sponsored research agreement between Emory University and Merck and Co., Bristol-Myers Squibb, Boerhinger-Ingelheim, and Vaccinex. Dr. El-Rayes has been on the advisory board for Ipsen, Natera, AstraZeneca, Bristol-Myers Squibb, Inc., been on the Data Safety Monitoring Board for Exelixis and Erytech and been a consultant to Merck and Co. and receives compensation for these services. The terms of this arrangement have been reviewed and approved by Emory University in accordance with its conflict of interest policies. Dr. El-Rayes has received research funding through a sponsored research agreement between Emory University and Bristol-Myers Squibb, Boston Biomedical, Novartis, Merck and Co, Bayer, Exelixis, Pfizer, AstraZeneca, Xencor, and EUSA. Dr. Paulos has received research funding through a sponsored research agreement between the Medical University of South Carolina and Obsidian, Lycera, ThermoFisher and is the Co-Founder of Ares Immunotherapy. Dr. Carson is on the advisory board for Dragonfly. All other authors declare no conflicts of interest.

Data and Materials Availability: All data are available in the main text or the supplementary materials.

Ethics Approval: This study did not receive nor require ethics approval, as it does not involve human participants.

\section{Acknowledgements:}

We would like to acknowledge the cores at Winship Cancer Institute and Emory University that made this research possible including the Pediatric/Winship Flow Cytometry Core, the Winship Cancer Tissue and Pathology Shared Resource, the Winship Biostatistics and Bioinformatics Core, the Winship Cancer 
Animal Models Shared Resource and the Winship Integrated Cellular Imaging Core and the Emory Integrated Genomics Core (EIGC), under NIH/NCl award number P30CA138292. The content is solely the responsibility of the authors and does not necessarily represent the official views of the National Institutes of Health.

\section{References}

1. Ottaviano M, De Placido S, Ascierto PA. Recent success and limitations of immune checkpoint inhibitors for cancer: a lesson from melanoma. Virchows Arch 2019;474(4):421-32. doi: 10.1007/s00428019-02538-4 [published Online First: 2019/02/13]

2. Tan E, El-Rayes B. Pancreatic Cancer and Immunotherapy: Resistance Mechanisms and Proposed Solutions. J Gastrointest Cancer 2019;50(1):1-8. doi: 10.1007/s12029-018-0179-z [published Online First: 2018/11/18]

3. Blando J, Sharma A, Higa MG, et al. Comparison of immune infiltrates in melanoma and pancreatic cancer highlights VISTA as a potential target in pancreatic cancer. Proc Natl Acad Sci U S A 2019;116(5):1692-97. doi: 10.1073/pnas.1811067116 [published Online First: 2019/01/13]

4. Ene-Obong A, Clear AJ, Watt $J$, et al. Activated pancreatic stellate cells sequester CD8+ $T$ cells to reduce their infiltration of the juxtatumoral compartment of pancreatic ductal adenocarcinoma. Gastroenterology 2013;145(5):1121-32. doi: 10.1053/j.gastro.2013.07.025 [published Online First: 2013/07/31]

5. Gorchs L, Fernandez Moro C, Bankhead P, et al. Human Pancreatic Carcinoma-Associated Fibroblasts Promote Expression of Co-inhibitory Markers on CD4(+) and CD8(+) T-Cells. Front Immunol 2019;10:847. doi: 10.3389/fimmu.2019.00847 [published Online First: 2019/05/10]

6. Lakins MA, Ghorani E, Munir $\mathrm{H}$, et al. Cancer-associated fibroblasts induce antigen-specific deletion of CD8 (+) T Cells to protect tumour cells. Nat Commun 2018;9(1):948. doi: 10.1038/s41467-018-03347-0 [published Online First: 2018/03/07]

7. Feig C, Jones JO, Kraman M, et al. Targeting CXCL12 from FAP-expressing carcinoma-associated fibroblasts synergizes with anti-PD-L1 immunotherapy in pancreatic cancer. Proc Natl Acad Sci U S A 2013;110(50):20212-7. doi: 10.1073/pnas.1320318110 [published Online First: 2013/11/28]

8. Farren MR, Mace TA, Geyer S, et al. Systemic Immune Activity Predicts Overall Survival in TreatmentNaive Patients with Metastatic Pancreatic Cancer. Clin Cancer Res 2016;22(10):2565-74. doi:

10.1158/1078-0432.CCR-15-1732 [published Online First: 2016/01/01]

9. Dienz O, Rincon M. The effects of IL-6 on CD4 T cell responses. Clin Immunol 2009;130(1):27-33. doi: 10.1016/j.clim.2008.08.018 [published Online First: 2008/10/11] 
10. Liu Q, Yu S, Li A, et al. Targeting interlukin-6 to relieve immunosuppression in tumor microenvironment. Tumour Bio/ 2017;39(6):1010428317712445. doi: 10.1177/1010428317712445 [published Online First: 2017/06/24]

11. Boj SF, Hwang Cl, Baker LA, et al. Organoid models of human and mouse ductal pancreatic cancer. Cel/ 2015;160(1-2):324-38. doi: 10.1016/j.cell.2014.12.021 [published Online First: 2015/01/06]

12. Chen $\mathrm{H}$, Liakou $\mathrm{Cl}$, Kamat $\mathrm{A}$, et al. Anti-CTLA-4 therapy results in higher CD4+ICOShi T cell frequency and IFN-gamma levels in both nonmalignant and malignant prostate tissues. Proc Natl Acad Sci U S A 2009;106(8):2729-34. doi: 10.1073/pnas.0813175106 [published Online First: 2009/02/10]

13. Liakou $\mathrm{Cl}$, Kamat A, Tang DN, et al. CTLA-4 blockade increases IFNgamma-producing CD4+ICOShi cells to shift the ratio of effector to regulatory T cells in cancer patients. Proc Natl Acad Sci US A 2008;105(39):14987-92. doi: 10.1073/pnas.0806075105 [published Online First: 2008/09/27]

14. Bengsch F, Knoblock DM, Liu A, et al. CTLA-4/CD80 pathway regulates T cell infiltration into pancreatic cancer. Cancer Immunol Immunother 2017;66(12):1609-17. doi: 10.1007/s00262-017-2053-4 [published Online First: 2017/09/01]

15. Diehl S, Chow CW, Weiss L, et al. Induction of NFATc2 expression by interleukin 6 promotes $T$ helper type 2 differentiation. J Exp Med 2002;196(1):39-49. doi: 10.1084/jem.20020026 [published Online First: 2002/07/03]

16. Diehl S, Rincon M. The two faces of IL-6 on Th1/Th2 differentiation. Mol Immunol 2002;39(9):531-6. doi: 10.1016/s0161-5890(02)00210-9 [published Online First: 2002/11/15]

17. Rincon M, Anguita J, Nakamura T, et al. Interleukin (IL)-6 directs the differentiation of IL-4-producing CD4+ T cells. J Exp Med 1997;185(3):461-9. doi: 10.1084/jem.185.3.461 [published Online First: 1997/02/03]

18. Oosterwegel MA, Mandelbrot DA, Boyd SD, et al. The role of CTLA-4 in regulating Th2 differentiation. J Immunol 1999;163(5):2634-9. [published Online First: 1999/08/24]

19. Paterson AM, Lovitch SB, Sage PT, et al. Deletion of CTLA-4 on regulatory T cells during adulthood leads to resistance to autoimmunity. J Exp Med 2015;212(10):1603-21. doi: 10.1084/jem.20141030 [published Online First: 2015/09/16]

20. Mace TA, Ameen Z, Collins A, et al. Pancreatic cancer-associated stellate cells promote differentiation of myeloid-derived suppressor cells in a STAT3-dependent manner. Cancer Res 2013;73(10):3007-18. doi: 10.1158/0008-5472.can-12-4601 [published Online First: 2013/03/22]

21. McLoughlin RM, Jenkins BJ, Grail D, et al. IL-6 trans-signaling via STAT3 directs T cell infiltration in acute inflammation. Proc Natl Acad Sci U S A 2005;102(27):9589-94. doi: 10.1073/pnas.0501794102 [published Online First: 2005/06/25] 
22. Loetscher M, Gerber B, Loetscher P, et al. Chemokine receptor specific for IP10 and mig: structure, function, and expression in activated T-lymphocytes. J Exp Med 1996;184(3):963-9. doi:

10.1084/jem.184.3.963 [published Online First: 1996/09/01]

23. Loetscher $\mathrm{M}$, Loetscher $\mathrm{P}, \mathrm{Brass} \mathrm{N}$, et al. Lymphocyte-specific chemokine receptor $\mathrm{CXCR3}$ : regulation, chemokine binding and gene localization. Eur J Immuno/ 1998;28(11):3696-705. doi:

10.1002/(SICI)1521-4141(199811)28:11<3696::AID-IMMU3696>3.0.C0;2-W [published Online First: 1998/12/08]

24. Cole KE, Strick CA, Paradis TJ, et al. Interferon-inducible T cell alpha chemoattractant (I-TAC): a novel non-ELR CXC chemokine with potent activity on activated $T$ cells through selective high affinity binding to CXCR3. J Exp Med 1998;187(12):2009-21. doi: 10.1084/jem.187.12.2009 [published Online First: 1998/06/24]

25. Lu B, Humbles A, Bota D, et al. Structure and function of the murine chemokine receptor CXCR3. Eur J Immuno/ 1999;29(11):3804-12. doi: 10.1002/(SICI)1521-4141(199911)29:11<3804::AID-

IMMU3804>3.0.C0;2-9 [published Online First: 1999/11/11]

26. Mace TA, Shakya R, Pitarresi JR, et al. IL-6 and PD-L1 antibody blockade combination therapy reduces tumour progression in murine models of pancreatic cancer. Gut 2016 doi: 10.1136/gutjnl-2016-311585 [published Online First: 2016/11/01]

27. Angevin E, Tabernero J, Elez E, et al. A phase I/II, multiple-dose, dose-escalation study of siltuximab, an anti-interleukin- 6 monoclonal antibody, in patients with advanced solid tumors. Clin Cancer Res 2014;20(8):2192-204. doi: 10.1158/1078-0432.CCR-13-2200 [published Online First: 2014/02/25]

28. Kamath SD, Kalyan A, Kircher S, et al. Ipilimumab and Gemcitabine for Advanced Pancreatic Cancer: A Phase Ib Study. Oncologist 2019 doi: 10.1634/theoncologist.2019-0473 [published Online First: 2019/11/20]

29. Royal RE, Levy C, Turner K, et al. Phase 2 trial of single agent Ipilimumab (anti-CTLA-4) for locally advanced or metastatic pancreatic adenocarcinoma. J Immunother 2010;33(8):828-33. doi: 10.1097/CJI.0b013e3181eec14c [published Online First: 2010/09/16]

30. Lamano JB, Lamano JB, Li YD, et al. Glioblastoma-Derived IL6 Induces Immunosuppressive Peripheral Myeloid Cell PD-L1 and Promotes Tumor Growth. Clin Cancer Res 2019;25(12):3643-57. doi: 10.1158/1078-0432.CCR-18-2402 [published Online First: 2019/03/03]

31. Li J, Xu J, Yan X, et al. Targeting Interleukin-6 (IL-6) Sensitizes Anti-PD-L1 Treatment in a Colorectal Cancer Preclinical Model. Med Sci Monit 2018;24:5501-08. doi: 10.12659/MSM.907439 [published Online First: 2018/08/09] 
32. Tsukamoto H, Fujieda K, Miyashita A, et al. Combined Blockade of IL6 and PD-1/PD-L1 Signaling Abrogates Mutual Regulation of Their Immunosuppressive Effects in the Tumor Microenvironment. Cancer Res 2018;78(17):5011-22. doi: 10.1158/0008-5472.CAN-18-0118 [published Online First: 2018/07/04]

33. Biffi G, Oni TE, Spielman B, et al. IL1-Induced JAK/STAT Signaling Is Antagonized by TGFbeta to Shape CAF Heterogeneity in Pancreatic Ductal Adenocarcinoma. Cancer Discov 2019;9(2):282-301. doi: 10.1158/2159-8290.CD-18-0710 [published Online First: 2018/10/28]

34. Ohlund D, Handly-Santana A, Biffi G, et al. Distinct populations of inflammatory fibroblasts and myofibroblasts in pancreatic cancer. J Exp Med 2017;214(3):579-96. doi: 10.1084/jem.20162024 [published Online First: 2017/02/25]

35. Jang JE, Hajdu CH, Liot C, et al. Crosstalk between Regulatory T Cells and Tumor-Associated Dendritic Cells Negates Anti-tumor Immunity in Pancreatic Cancer. Cell Rep 2017;20(3):558-71. doi:

10.1016/j.celrep.2017.06.062 [published Online First: 2017/07/21]

36. Alspach E, Lussier DM, Miceli AP, et al. MHC-II neoantigens shape tumour immunity and response to immunotherapy. Nature 2019;574(7780):696-701. doi: 10.1038/s41586-019-1671-8 [published Online First: 2019/10/28]

37. Dunn GP, Koebel CM, Schreiber RD. Interferons, immunity and cancer immunoediting. Nat Rev Immunol 2006;6(11):836-48. doi: 10.1038/nri1961 [published Online First: 2006/10/26]

38. Ikeda H, Old LJ, Schreiber RD. The roles of IFN gamma in protection against tumor development and cancer immunoediting. Cytokine Growth Factor Rev 2002;13(2):95-109. doi: 10.1016/s13596101(01)00038-7 [published Online First: 2002/03/20]

39. Detjen KM, Farwig K, Welzel M, et al. Interferon gamma inhibits growth of human pancreatic carcinoma cells via caspase-1 dependent induction of apoptosis. Gut 2001;49(2):251-62. doi: 10.1136/gut.49.2.251 [published Online First: 2001/07/17]

40. Chin YE, Kitagawa M, Kuida K, et al. Activation of the STAT signaling pathway can cause expression of caspase 1 and apoptosis. Mol Cell Biol 1997;17(9):5328-37. doi: 10.1128/mcb.17.9.5328 [published Online First: 1997/09/01]

41. Gao J, Shi LZ, Zhao H, et al. Loss of IFN-gamma Pathway Genes in Tumor Cells as a Mechanism of Resistance to Anti-CTLA-4 Therapy. Cel/ 2016;167(2):397-404 e9. doi: 10.1016/j.cell.2016.08.069 [published Online First: 2016/09/27]

42. Chow MT, Ozga AJ, Servis RL, et al. Intratumoral Activity of the CXCR3 Chemokine System Is Required for the Efficacy of Anti-PD-1 Therapy. Immunity 2019;50(6):1498-512 e5. doi:

10.1016/j.immuni.2019.04.010 [published Online First: 2019/05/18] 
43. Uemura M, Trinh VA, Haymaker C, et al. Selective inhibition of autoimmune exacerbation while preserving the anti-tumor clinical benefit using IL- 6 blockade in a patient with advanced melanoma and Crohn's disease: a case report. J Hematol Oncol 2016;9(1):81. doi: 10.1186/s13045-016-0309-7 [published Online First: 2016/09/07]

44. Maude SL, Barrett D, Teachey DT, et al. Managing cytokine release syndrome associated with novel T cell-engaging therapies. Cancer J 2014;20(2):119-22. doi: 10.1097/PPO.0000000000000035 [published Online First: 2014/03/29]

45. Horisberger A, La Rosa S, Zurcher JP, et al. A severe case of refractory esophageal stenosis induced by nivolumab and responding to tocilizumab therapy. J Immunother Cancer 2018;6(1):156. doi: 10.1186/s40425-018-0481-0 [published Online First: 2018/12/28]

46. Bankhead P, Loughrey MB, Fernandez JA, et al. QuPath: Open source software for digital pathology image analysis. Sci Rep 2017;7(1):16878. doi: 10.1038/s41598-017-17204-5 [published Online First: 2017/12/06]

47. Muranski P, Boni A, Antony PA, et al. Tumor-specific Th17-polarized cells eradicate large established melanoma. Blood 2008;112(2):362-73. doi: 10.1182/blood-2007-11-120998 [published Online First: 2008/03/21]

\section{Figures}


Figure 1.
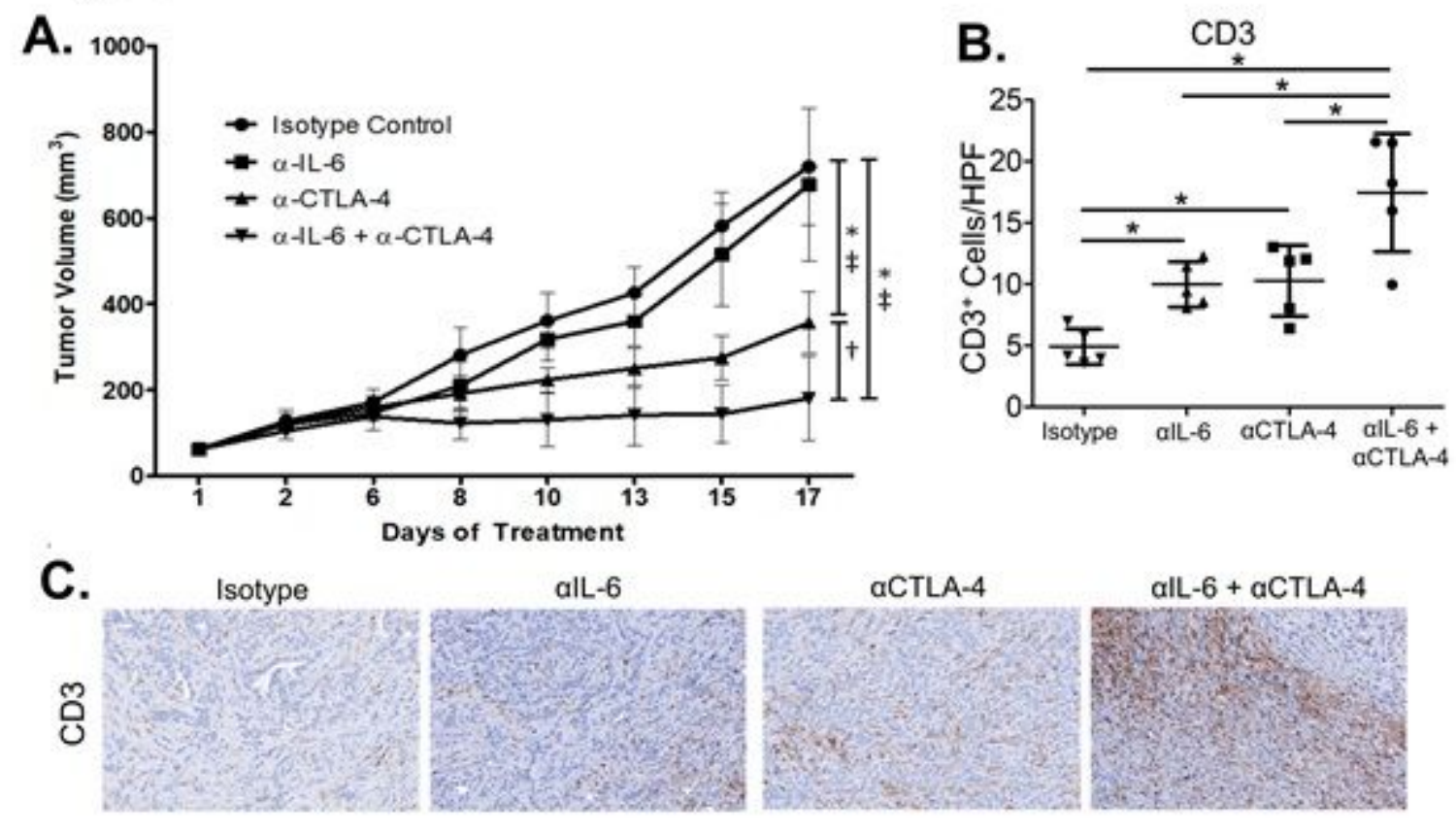

aCTLA-4

alL-6 + aCTLA-4
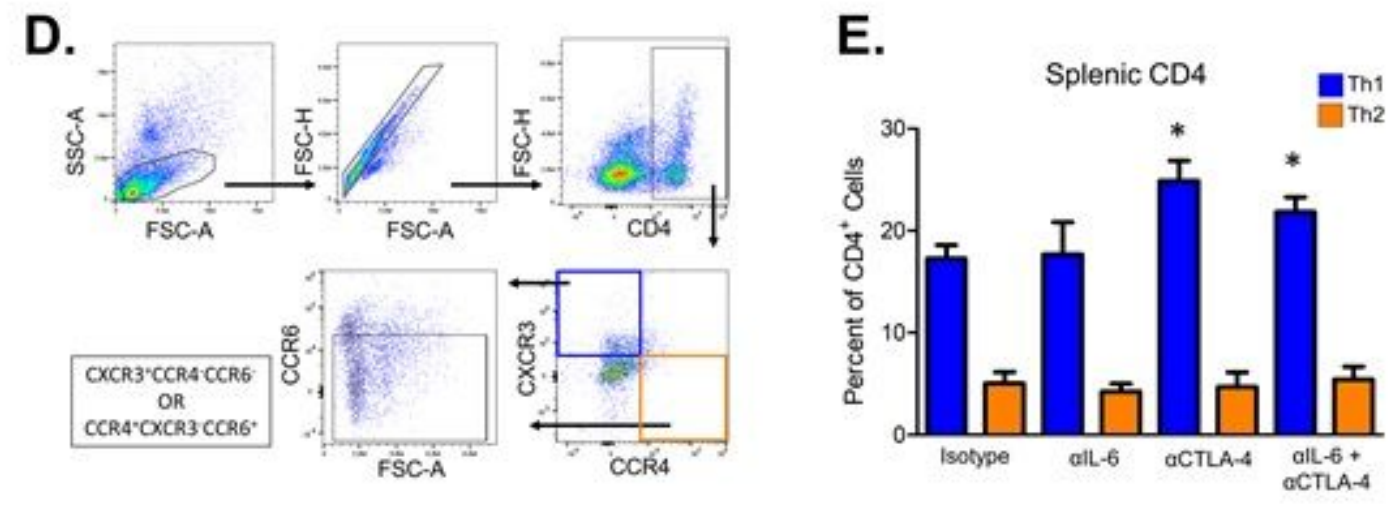

\section{Figure 1}

Combined blockade of IL- 6 and CTLA-4 significantly inhibits tumor growth and promotes CD8 T cell infiltration of tumors in a subcutaneous murine model of pancreatic cancer. MT5 murine pancreatic tumor cells were subcutaneously injected into C57BL/ 6 mice with treatment beginning when tumors reached $50-100 \mathrm{~mm} 3$. Mice were treated with $200 \mathrm{mg}$ (intraperitoneal injection 3 times/week) of isotype control, cytokine blockade (anti-IL-6) and/or anti-CTLA-4 antibodies ( $\mathrm{n}=5$ mice/group) until mice met prespecified IACUC-approved early removal criteria. (A) Changes in tumor volume as determined by caliper measurement throughout the course of antibody treatment. Mean $\pm S D ; * P<0.05$ vs. Isotype Control, $\neq$ $P<0.05$ vs. Anti-IL-6, $+P<0.05$ vs. Anti-CTLA-4. (B) Mean \pm SD for percent of cells expressing CD $3+$ in subcutaneous tumors per high-powered field. Symbols represent individual mice; * indicates significance compared to isotype control treated mice. P-values for significant comparisons are as follows: Isotype vs alL-6 (0.0013), Isotype vs aCTLA-4 (0.0058) and Isotype vs alL-6+aCTLA-4 (0.0058) (C) Representative 20x images of IHC staining for CD3 in FFPE tumor tissue slices from mice in the different treatment groups. (D) Splenocytes were isolated from the mice receiving treatment as stated in figure $1 \mathrm{~A}$. Flow 
cytometry was performed with antibodies against CD4, CCR6, CXCR3, CCR4, and RORyt. CD4+CCR6CXCR3+CCR4- were identified as suggestive of a Th1 phenotype and CD4+CCR6- CXCR3-CCR4+ as a Th2 phenotype. (E) Graph of mean percentages of CD4+ T cells that have a Th1 or Th2 phenotype. Data shown as Mean $\pm \mathrm{SD}$; * indicates significance compared to isotype control treated mice. P-values for significant comparisons are as follows: Isotype vs aCTLA-4 (<0.0001), Isotype vs alL-6+aCTLA-4 (0.0004), alL-6 vs aCTLA-4 (0.0026), alL-6 vs alL-6+aCTLA-4 (0.0278).

Figure 2.
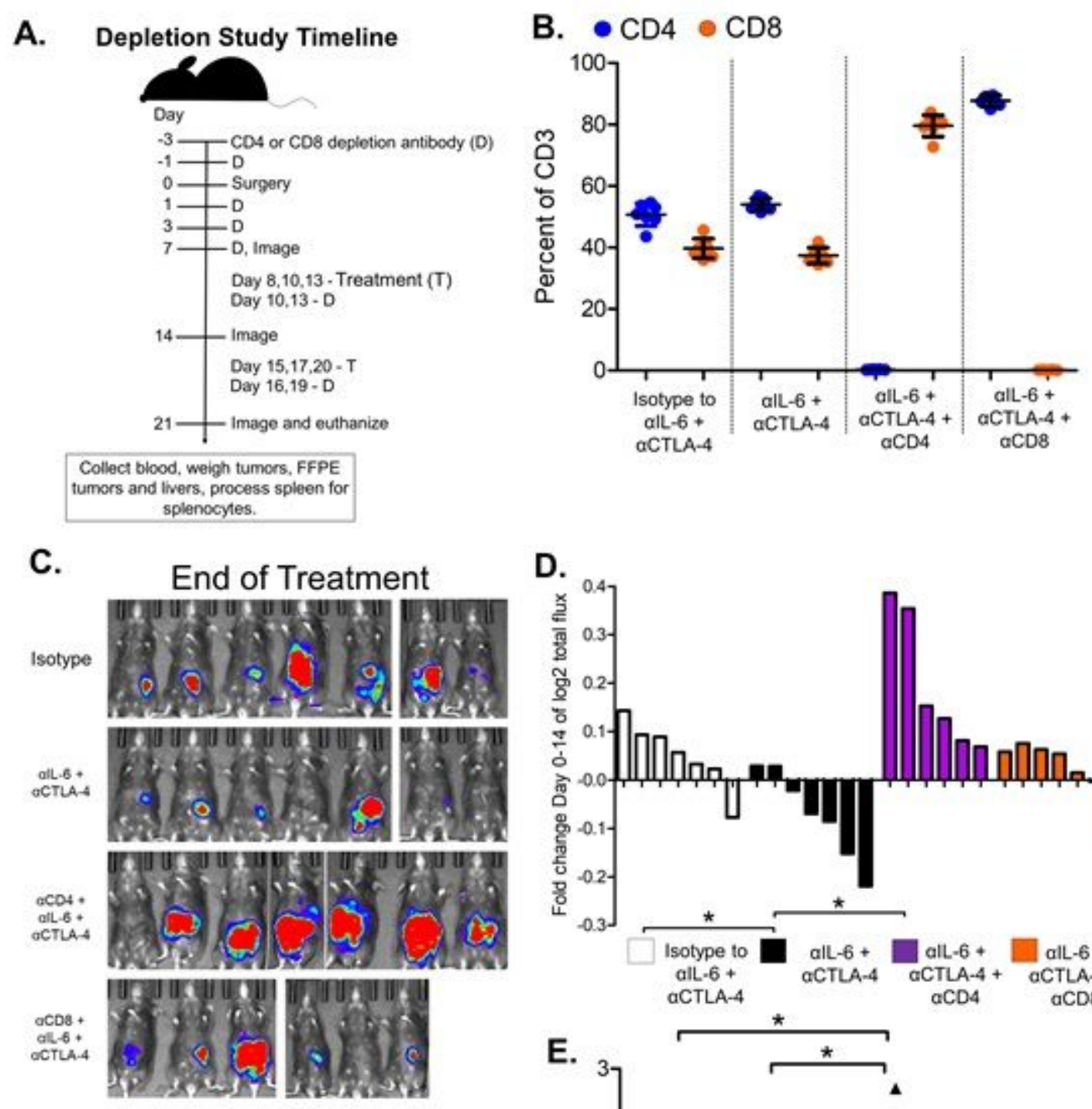

D.
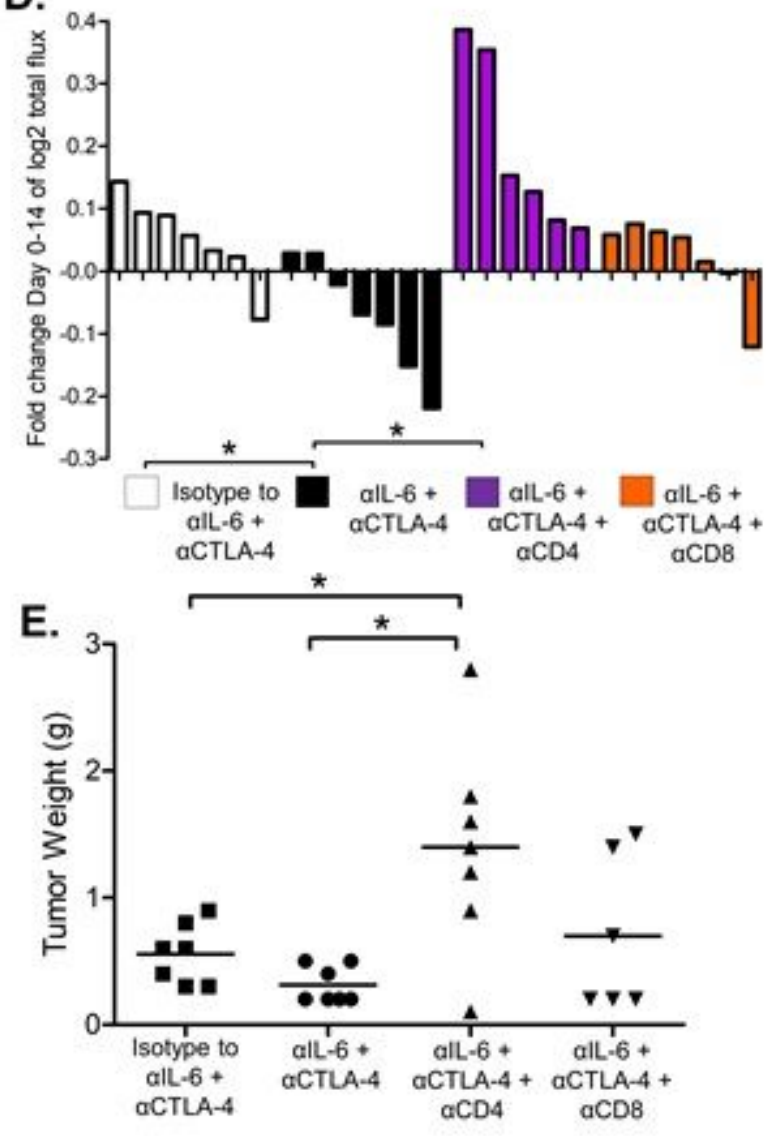

Figure 2 
Both CD4+ and CD8+ T cells are required for anti-tumor responses to orthotopic pancreatic tumors in mice treated with combined IL- 6 and CTLA- 4 blockade. Female C57BL/ 6 mice, 6-8 weeks of age were orthotopically injected with $2 \times 105 \mathrm{KPC}$-luc cancer cells and imaged 1 week later by bioluminescent imaging (BLI) to confirm tumor establishment. (A) Timeline for the administration of CD4 or CD8 depleting antibodies relative to orthotopic injection and subsequent administration of IL-6 and CTLA-4 blocking antibodies or isotype control antibodies. (B) At the study endpoint, mice were euthanized and spleens were collected and processed for isolation of splenocytes. Splenocytes were then stained for CD3, CD 4 and CD8 markers. The graph demonstrates the percentage of CD3+ cells in splenocytes from each group that expressed the markers CD4 (blue) or CD8 (orange). (C) End of treatment bioluminescent images for each mouse in the study outlined in Figure 2A are displayed. (D) Tumor growth for each mouse from the study outline in Figure 2A was measured over time by BLI and the fold change in Log2 of total flux for each mouse was graphed as a bar. P-values for significant comparisons are as follows: Isotype vs alL-6+aCTLA-4 (0.0166) and alL-6+aCTLA-4 vs alL-6+aCTLA-4+aCD4 (0.0020) (E) At the study endpoint (see outline in Figure 2A), mice were euthanized and the weight of each tumor was measured and graphed with symbols representing individual mice and mean displayed for each treatment group. Pvalues for significant comparisons are as follows: Isotype vs alL-6+aCTLA-4 (0.0397) and alL-6+aCTLA-4 vs alL-6+aCTLA-4+aCD4 (0.0144). 
Figure 3.
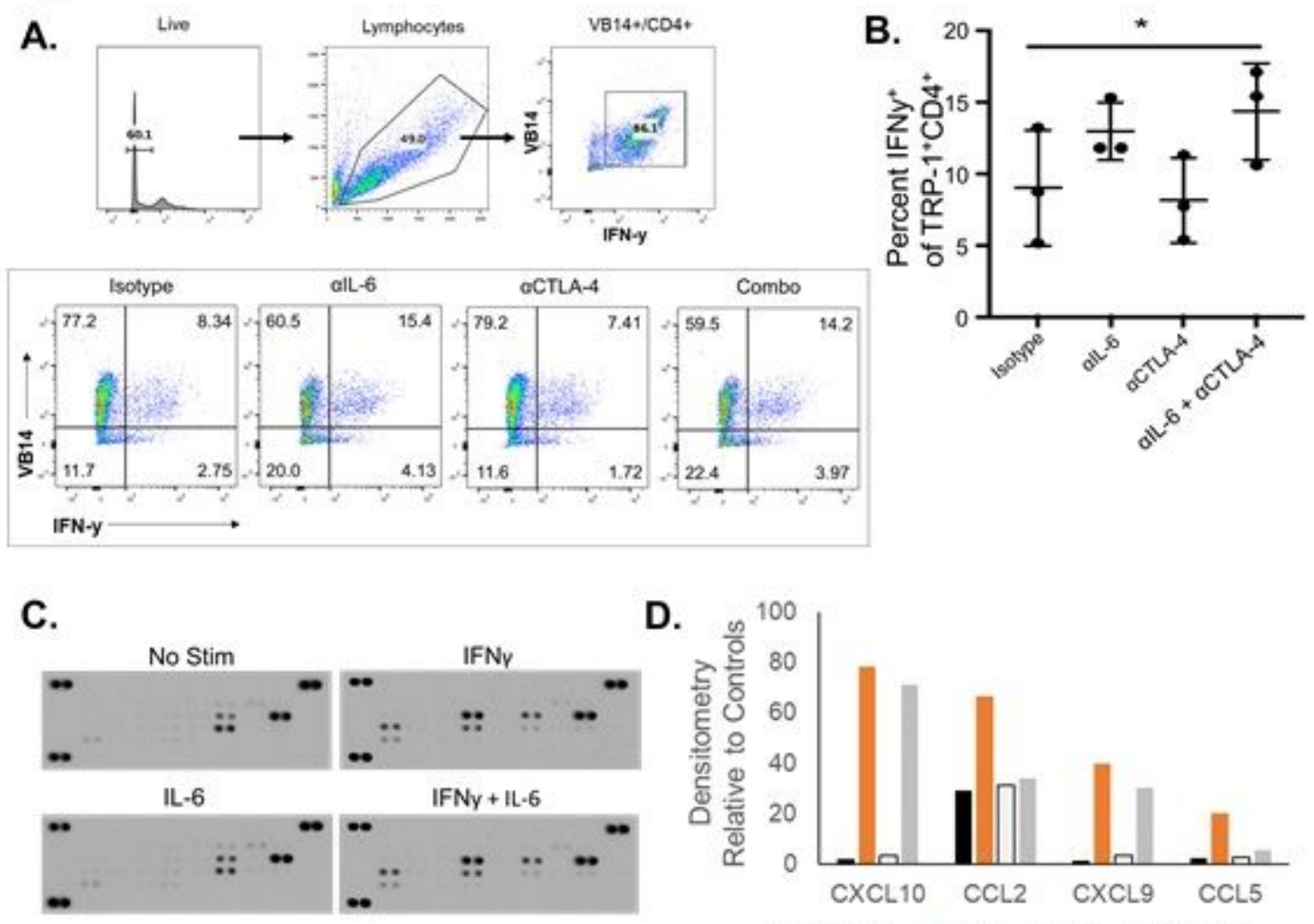

-No Stim =IFNy aIL-6 =IFNy+IL-6
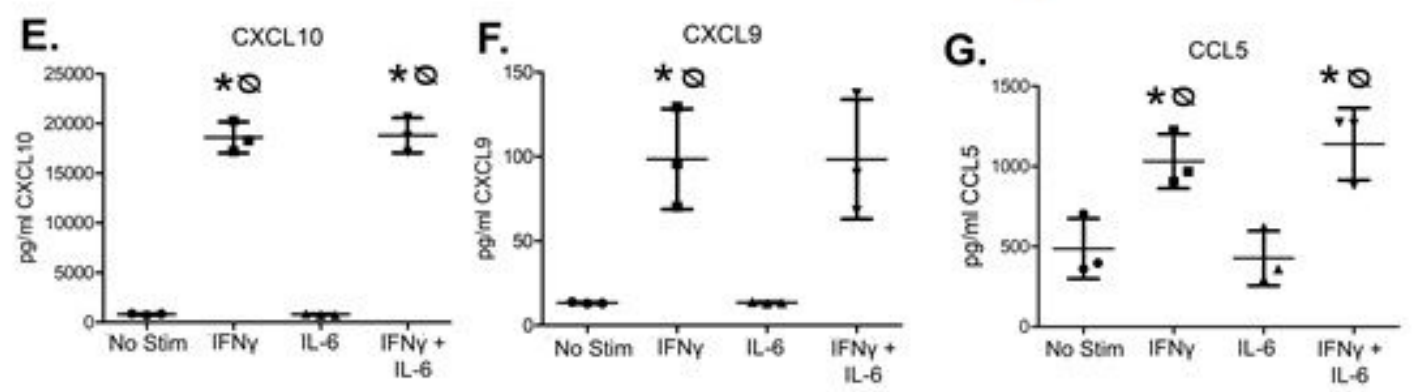

\section{Figure 3}

Combined blockade of IL- 6 and CTLA-4 promotes IFN-y production by antigen-activated CD4 T cells, which can elicit changes in chemokine production by pancreatic tumor cells. (A) Trp-1 specific CD4+ T cells were stimulated in the presence of antibodies to IL-6, CTLA-4, the combination of both or isotype control antibodies. The percentage of cells expressing V $\beta 14$ and IFN- $\gamma$ were quantified by flow cytometry. (B) Graph shows percentages of CD4+ T cells expressing IFN- $\gamma$ for each mouse with mean $\pm S D$. CD 4+ T cells expressed more IFN- $\gamma$ in the presence of dual CTLA- 4 and IL- 6 blockade compared to isotype control antibodies $(p=0.0217)(C)$ KPC-luc cells were plated at $2 \times 105$ cells per well in 6 well plates and then stimulated with $1 \mu \mathrm{g} / \mathrm{ml} \mathrm{IFNy,} 10 \mathrm{ng} / \mathrm{ml} \mathrm{IL-6}$, both or vehicle control for $24 \mathrm{hrs}$. Resulting supernatants were collected and analyzed using the Proteome Profiler Mouse Chemokine Array Kit. Shown are resulting images of a chemokine membrane exposed to supernatants of KPC-luc cells from each treatment condition. (D) The relative densitometry to loading controls for CXCL10, CCL2, CXCL9, CCL5 as detected by the chemokine array are graphed for each treatment condition. Repetitions of the experiment described 
in Figure $3 \mathrm{C}$ were quantified by ELISA and the resulting concentrations were graphed for (E) CXCL10 (F) CXCL9 and (G) CCL5. Data are shown with symbols marking individual experiments and mean \pm SD for

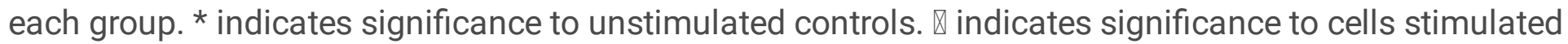
only with IL-6. P-values for significant comparisons are listed in supplementary table 2.

Figure 4.
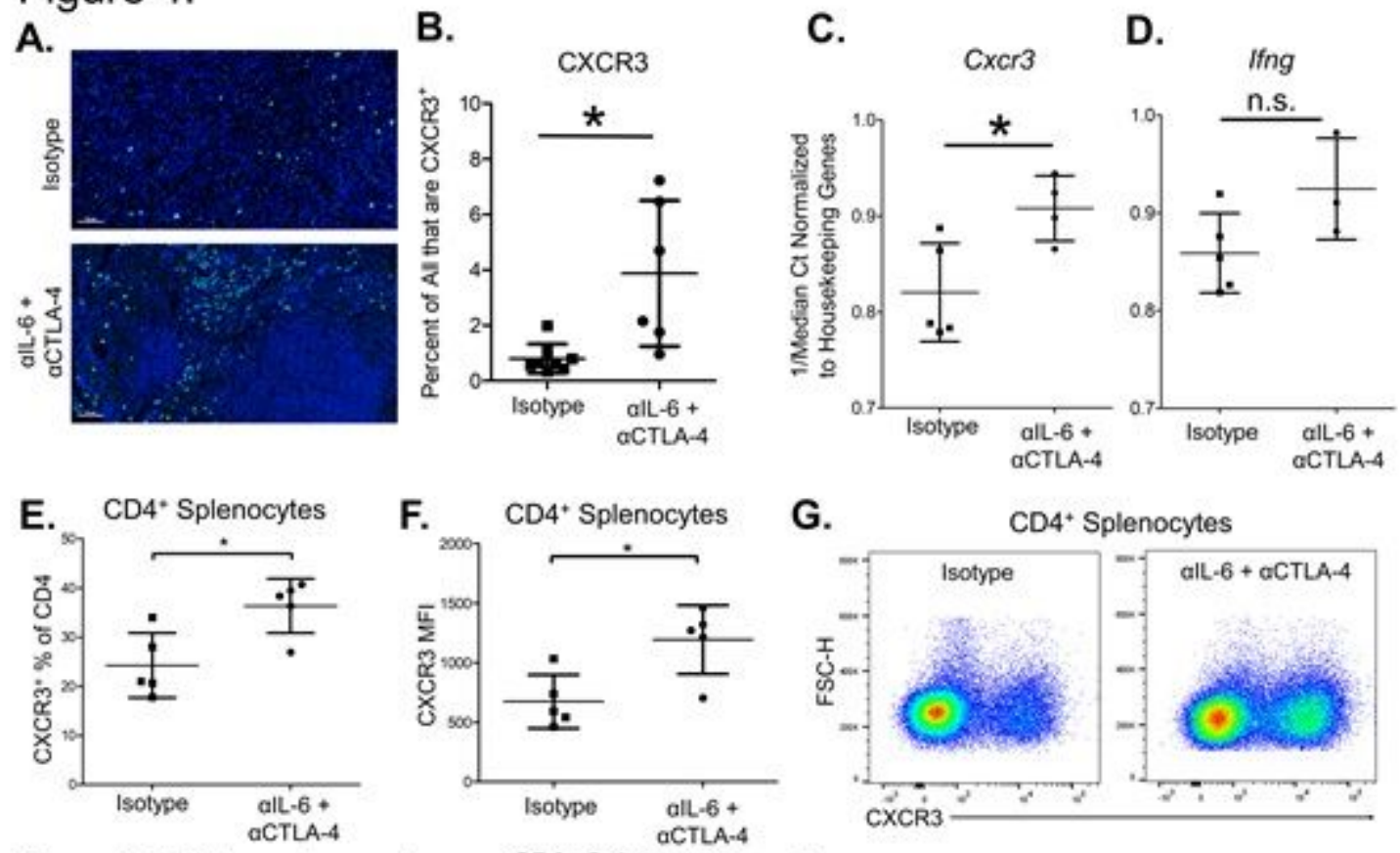

H. $\mathrm{CD}^{*}$ Splenocytes
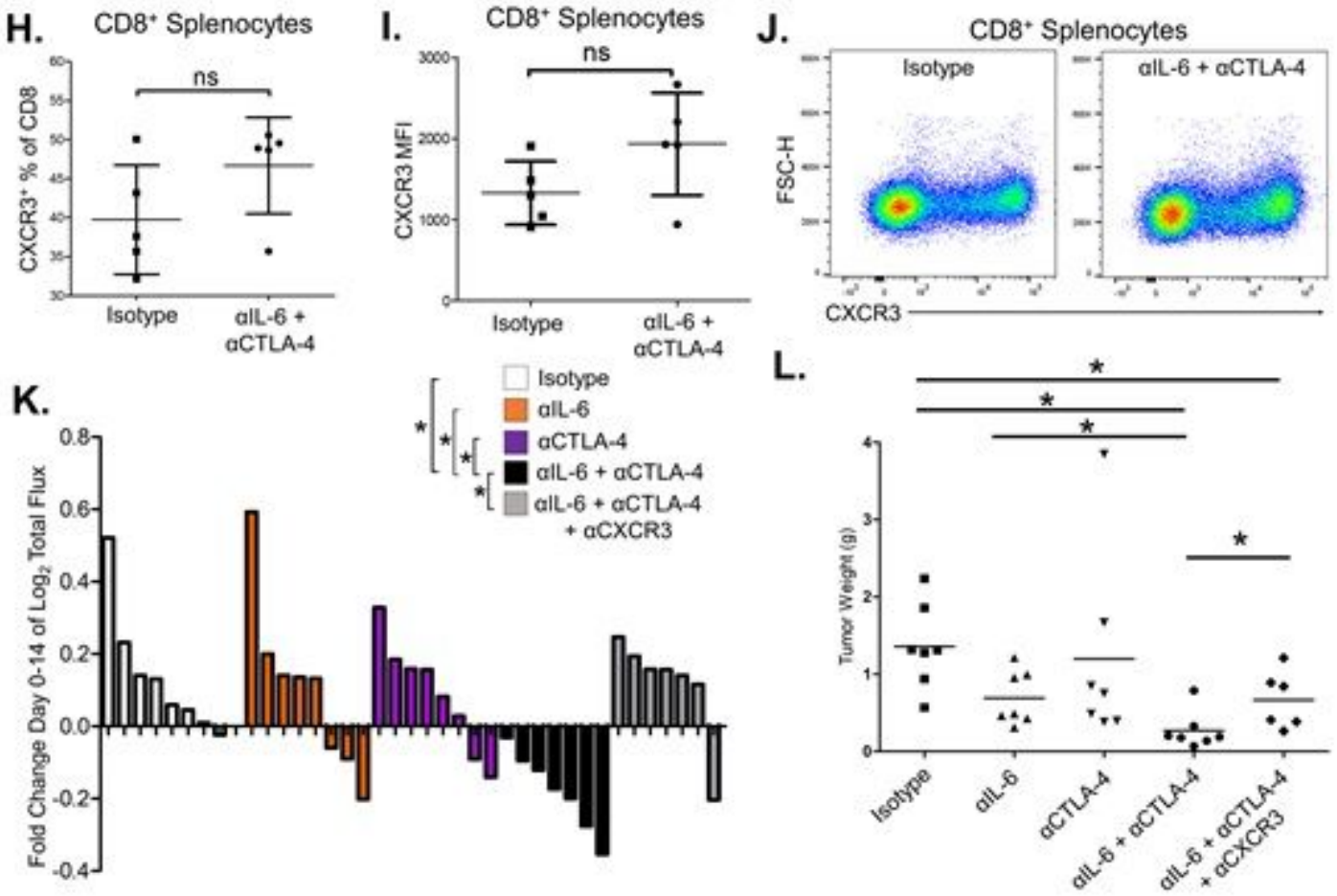

\section{Figure 4}

Treatment of murine orthotopic pancreatic tumors with antibodies to IL- 6 and CTLA-4 results in significant tumor regression and increased intra-tumoral CD4+ and CD8+ T cells in a CXCR3 dependent manner. C57BL/ 6 mice were orthotopically injected with 2×105 KPC-luc cancer cells and treated 3 times a 
week for 2 weeks with antibodies to IL-6 and CTLA-4 or isotype control antibodies. (A) FFPE tumors were stained for DAPI (blue) and CXCR3 (green) by IHC and imaged using a Vectra Polaris immunofluorescent slide scanner (B) Whole slide scans were analyzed using Qupath and the percentage of all cells positive for $\mathrm{CXCR3}$ is graphed. Each symbol represents a unique mouse. * indicates significance $(p=0.0371)$. (C) CD3+CD 45+ TIL were enriched from tumors of mice treated with antibodies to IL- 6 and CTLA-4 or isotype control antibodies. RNA was isolated and analyzed by PCR for the expression of Cxcr3 or (D) Ifng. Median Ct values were normalized to housekeeping genes and plotted as indicated. * indicates significance $(p=0.0223)$. ( $E)$ Splenocytes from these mice were stained for CD3, CD4, CD8 and CXCR3. The percentage of CD4+ T cells expressing CXCR3 and $(F)$ the MFI of CXCR3 on CD4+ T cells is graphed with $(G)$ representative flow plots of CXCR3 staining shown. $(H)$ The percentage of $C D 8+T$ cells expressing CXCR3 and (I) the MFI of CXCR3 on CD8+ T cells is graphed with $(\mathrm{J})$ representative flow plots of CXCR3 staining shown. * indicates significance. CXCR3+\% of CD4 ( $p=0.0132)$. CXCR3 MFI on CD4+ T cells $(p=0.0430)$. (K) Mice bearing orthotopic KPC-luc tumors were treated with antibodies to IL-6, CTLA-4, the combiantion, the combination and antibodies to $\mathrm{CXCR3}$, or isotype controls. Tumor growth was tracked by BLI and the Log2 fold change in total flux for each mouse was graphed as a bar. * indicates significance $(p<0.05)$ to mice receiving dual blockade of IL-6 and CTLA-4. P-values for significant comparisons are as follows: Isotype vs alL-6+aCTLA-4 (0.0003), alL-6 vs alL-6+aCTLA-4 (0.0104), aCTLA4 vs alL-6+aCTLA-4 (0.0039) and alL-6+aCTLA-4 vs alL-6+aCTLA-4+aCXCR3 (0.0029). (L) The total weight of each tumor was collected and graphed as mean for each treatment group. Symbols represent individual mice. * indicates significance $(p<0.05)$. P-values for significant comparisons are as follows: Isotype vs alL-6+aCTLA-4 (0.0005), alL-6 vs alL-6+aCTLA-4 (0.0224), Isotype vs IL-6+aCTLA-4+aCXCR3 (0.0247) and alL-6+aCTLA-4 vs alL-6+aCTLA-4+aCXCR3 (0.0405). 
Figure 5.

A.

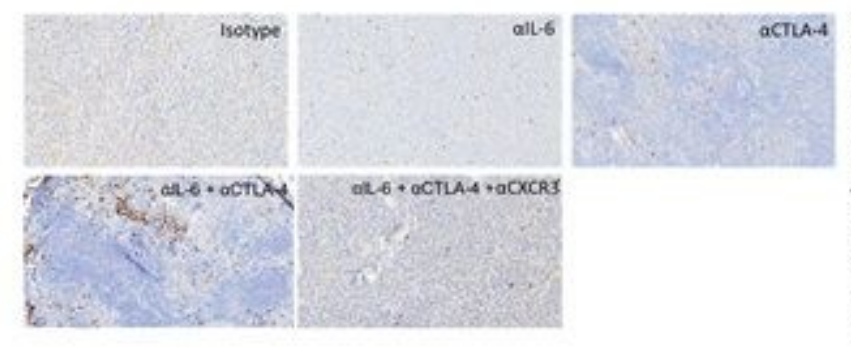

B.

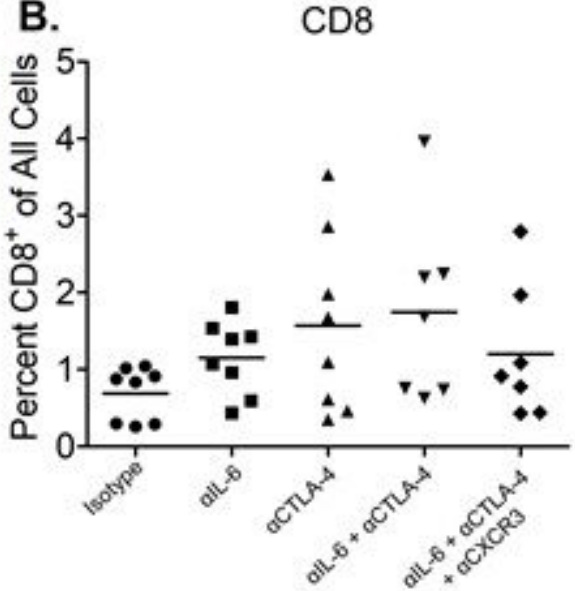

C.

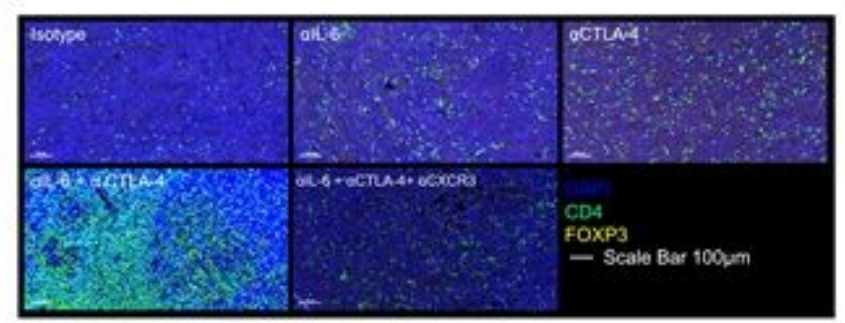

D.

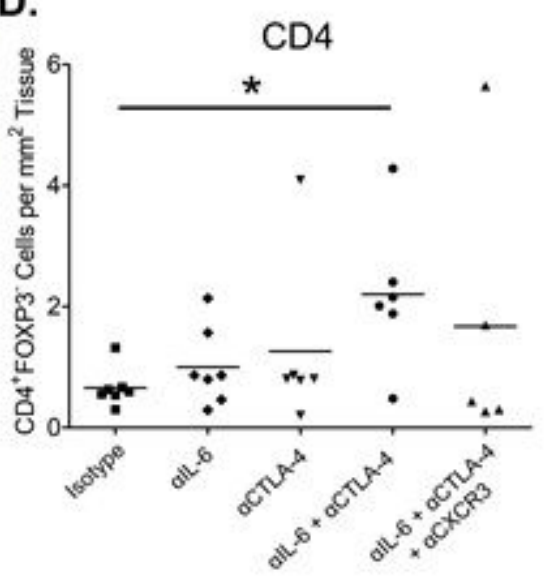

\section{Figure 5}

T cell infiltration of pancreatic tumors in altered in the presence of combined IL- 6 and CTLA-4 blockade. FFPE tissue slices of tumors from mice described in Figure 4 were stained for CD8 by IHC. (A) Representative images of IHC staining for CD8 in tumors from mice. (B) Infiltrating CD8 T cells were quantified using Qupath and graphed as the percentage of cells expressing CD8. Results are displayed as mean for each treatment group. Symbols represent individual mice. (C) FFPE Tissue slices from these tumors were also stained for CD 4 and FOXP3 with DAPI counterstain followed by primary antibodydetection with Opal-conjugated antibodies. Representative images are displayed with CD4 staining in green, FOXP3 staining in red and DAPI staining in blue. Scale bars are 100 $\mathrm{m}$. (D) After scanning slides using a Perkin Elmer Vectra Polaris fluorescent slide scanner, the percentage of cells positive for CD 4 but negative for FOXP3 were quantified with Qupath and graphed as mean for each treatment group. * indicates significant difference between isotype treated mice and mice receiving dual IL- 6 and CTLA-4 blockade $(p=0.0297)$. 
Figure 6.
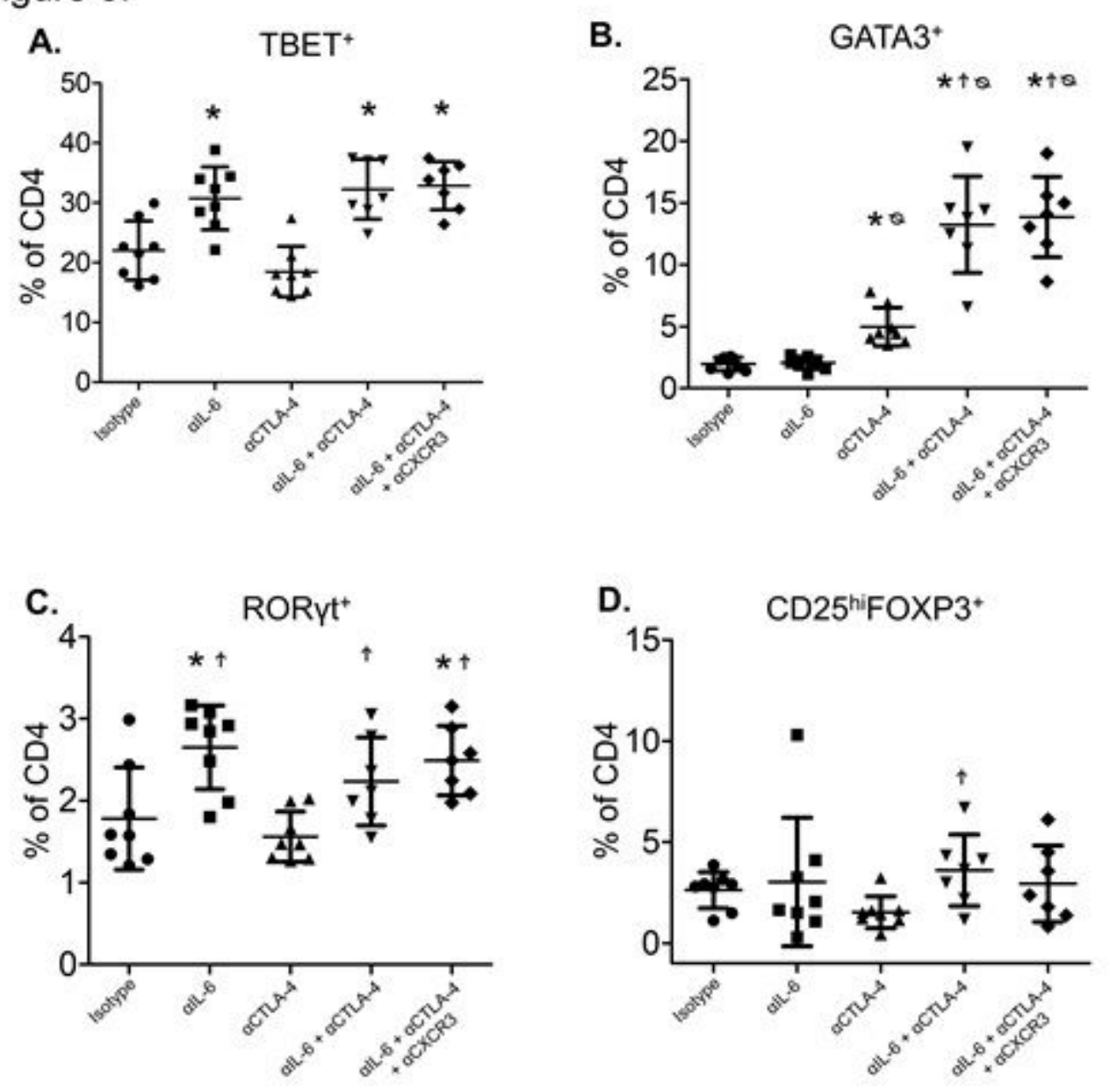

Figure 6

Combined blockade of IL- 6 and CTLA-4 in mice bearing orthotopic pancreatic tumors results in systemic changes in CD4-helper T cells. Splenocytes were isolated from the mice receiving treatment as stated in Figure 4. Cells were stained for CD3, CD4, and CD8 surface markers with Ghost 780 viability stain to mark dead cells. After fixation and permeabilization, cells were stained for the transcription factors TBET, GATA3, RORyt and FOXP3. The percentage of CD4+ T cells that were (A) TBET+ (B) GATA3+ (C) RORyt+ or (D) CD25hiFOXP3+ were graphed as mean \pm SD with symbols indicating significance $(p<0.05)$ compared to * isotype control mice, $\nabla$ aCTLA- 4 treated mice, or $\nabla$ alL- 6 treated mice. P-values for significant comparisons are listed in supplementary table 2 .

\section{Supplementary Files}

This is a list of supplementary files associated with this preprint. Click to download.

- SupplementaryFiguresandTables.pdf 